\title{
Analyzing the Performance of Spectrum Sensing Algorithms for IEEE 802.11 af Standard in Cognitive Radio Network ${ }^{*}$
}

\author{
Tanuja S. Dhope (Shendkar) ${ }^{1}$, Dina Simunic ${ }^{1}$, Antun Kerner ${ }^{2}$ \\ ${ }^{1}$ Faculty of Electrical Engineering and Computing, University of Zagreb, Croatia, \\ tanuja_dhope@yahoo.com,dina.simunic@fer.hr, \\ ${ }^{2}$ Ericsson-Nikola Tesla, Zagreb, Croatia, \\ antun.kerner@ericsson.com
}

\begin{abstract}
In the entire world the wireless communication systems are represented by 2G and 3G systems with all stages of evolution towards $4 \mathrm{G}$ systems. The complexity of wireless networks requires a careful design, especially related to bandwidth and energy efficiency. Bandwidth efficiency is very important parameter, because it relates to frequency spectrum, which is a natural limited resource. The cognitive radio has been proposed as the future technology to meet the ever increasing demand of the radio spectrum by allocating the spectrum dynamically to allow unlicensed access on noninterfering basis. The digital dividend of $700 \mathrm{MHz}$ band (mainly used by TV broadcast services) opens the door for cognitive radio applications due to its excellent propagation characteristics compared to GSM $1800 \mathrm{MHz}, 2.1 \mathrm{GHz}$ or 2.5 $\mathrm{GHz}$ bands. In cognitive radio, spectrum sensing is the fundamental problem. In this paper we are analyzing the performance of spectrum sensing algorithms; energy detection and covariance absolute value utilizing TV white space for IEEE 802.11 af standard.
\end{abstract}

Keywords: energy detection, covariance based detection, fading channels, spectrum sensing, cognitive radio, IEEE 802.11af.

\section{Introduction}

Although spectrum is seen as a scarce natural resource, measurements show that often there are moments in time and space of the nonutilized spectrum by the allocated services. Therefore, it can be said that the spectrum is being used inefficiently [1], thus demanding dynamic allocation of spectrum instead of static [2][3]. Recently, there have been growing interests in cognitive radio, where secondary opportunistic radio exploits opportunistically spectrum left-overs- or so-called "White Spaces", by means of knowledge of the environment and cognition capability, and adapts their radio parameters accordingly, [2][3][4][5]. The digital dividend of $700 \mathrm{MHz}$ band (mainly used by TV broadcast services) opens the door for cognitive radio applications due to its excellent propagation characteristics compared to GSM $1800 \mathrm{MHz}, 2.1 \mathrm{GHz}$ or 2.5 $\mathrm{GHz}$ bands. The upcoming IEEE 802.11af standard (known as super-Wi-Fi) is based on cognitive radio (CR) [6], utilizing the bandwidth within TV broadcast stations [7] [8] [9] [10].

\footnotetext{
* A shorter version of this paper has been submitted to the 35th Jubilee International Convention MIPRO 2012, May 2012, under the title "Hybrid Detection Method for Spectrum Sensing in Cognitive Radio"[17] and further a modified version of this paper has also been submitted to the same scientific event under the title "Performance Analysis of Covariance Based Detection in Cognitive Radio”[19].
}

CR makes opportunistic use of the spectrum by allowing unlicensed or secondary user (SU) to reuse the spectrum whenever the licensed or primary user (PU) is inactive. The SUs are required to perform the frequent spectrum sensing for detecting the presence of PUs with a high probability of detection and vacant the channel or reduce transmit power. For IEEE 802.11af standard, time to vacate band after PUs detection is 2 s with $90 \%$ of probability of detection (Pd) and 10\% of probability of false alarm (Pfa) at Signal-to-Noise ratio (SNR) level as low as -20dB along with geo-location accuracy of $+/-50 \mathrm{~m}$ [6][9][10]. Sensing a spectrum is a crucial task in CR. There have been several sensing methods, including the likelihood ratio test (LRT) [11], energy detection (ED) method [11] [12] [13], matched filtering (MF)-based method [11] and cyclostationary detection method [11], each of which has different requirements and advantages or disadvantages. Although LRT is proven to be optimal, it is very difficult to use, because it requires exact channel information and distributions of the source signal and noise. To use LRT for detection, we need to obtain the channels and signal and noise distributions first, which are practically intractable. The MF-based method requires perfect knowledge of the channel responses from the primary user to the receiver and accurate synchronization (otherwise, its performance will dramatically be reduced). 
Energy detection method is a semi-blind detection which requires knowledge of noise power only for signal detection. In [12], sets of receiver operating characteristic (ROC) curves are drawn for several time-bandwidth products for spectrum sensing using energy detection but not specified exactly which type of signal is used. In [11], Blindly combined energy detection method which uses the spatial correlation of received signals based on energy detection, is analysed for independent and identically distributed source signal which is FM modulated wireless signal operated in vacant TV channels with a bandwidth less than $200 \mathrm{kHz}$, the flat-fading and the multipath fading channel. The experimental study for spectrum sensing in $2.4 \mathrm{GHz}$ ISM band over 85 $\mathrm{MHz}$ of bandwidth using energy detection for sine wave carrier and QPSK sensing is evaluated in [14]. In the same reference minimum detectable signal levels set by the receiver noise uncertainties are measured. The cyclostationary detection method needs to know the cyclic frequencies of the primary users, which may not be realistic for many spectrum reuse applications. Furthermore, this method demands excessive analogue-to-digital (A/D) converter requirements and signal processing capabilities [11]. In the references: [11] [15] [16], a new spectrum sensing algorithm based on covariance of the received signal called as Covariance Absolute Value (CAV) is proposed. CAV is a blind detection method, uses space-time signal correlation for signal detection which does not require any knowledge of noise and signal power. The covariances of signal and noise are generally different, which can be used in detection of PU.

But CAV method is very sensitive to signal correlation.

In [13], a new method called as 'Hybrid Detection method' is proposed which takes the advantage of ED and CAV by utilizing ED method in low correlation and CAV method in high correlation. Further the simulation results for hybrid detection method is studied in detail in [13] and [17].The hybrid detection method shows the better performance compared to energy detection and covariance method.

In [15] [16], the performance of ED and CAV is evaluated for Advanced Television Systems Committee (ATSC) of $6 \mathrm{MHz}$ bandwidth for the narrow-band signal of a wireless microphone, by analysis of multiple antennas . In [15], ROC curve is analyzed for wireless microphone signal for outdoor environment with Rician channel, further $P_{d}$ versus the smoothing factor is studied for a wireless microphone signal. Also in [15] $P_{d}$ versus different values of SNRs are studied for ATSC signal with single and multiple antennas which is concluded by the effect of time variant channel on the detection of ATSC signal. But in [15]-[16] the ROC curve and the effect of timevariant Rayleigh channel using ED and CAV spectrum sensing algorithms on detection of ATSC signal has not been studied. Further CAV method for DVB-T standard for $8 \mathrm{MHz}$ bandwidth has not been evaluated.

Since cognitive radio is an emerging technology, the performance of the spectrum sensing algorithm needs to be analyzed under conditions of different fading channels.

The goal of this paper is to present an analysis of energy detection and covariance based detection for DVB-T standard for $8 \mathrm{MHz}$ bandwidth in $2 \mathrm{k}$ mode used for mobile reception of standard television. This paper focuses on analyzing two algorithms under conditions of AWGN channel, Rayleigh and Rician fast and slow time variant fading channels using time-variant Jakes' model based on probability of detection under varying SNR conditions, $\mathrm{P}_{d}$ versus $\mathrm{P}_{f a}$ for different SNRs, $\mathrm{P}_{d}$ versus smoothing factor, $\mathrm{P}_{d}$ versus overall correlation coefficient and receiver operating characteristic (ROC) curve for DVB-T standard.

The organization of the paper is as follows. The overview of ED and CAV is made in section 2. Simulation is performed in section 3 for terrestrial digital video broadcasting based on the two methods, followed by conclusions in section 4 .

\section{Analysis of ED and CAV Methods}

The Neyman-Pearson criteria say that the spectrum detection is a binary hypothesis sensing problem [11] [12] [13].

$$
\begin{aligned}
& H_{0}: y(n)=\eta(n) \quad n=1,2,3, \ldots \\
& H_{1}: y(n)=s(n)+\eta(n) \quad n=1,2,3, \ldots
\end{aligned}
$$

where $s(n)$ indicates the PU and $\eta(n)$ is the additive white Gaussian noise which is assumed to be independent and identically distributed random variable with zero $\mathrm{n} \sigma_{\eta}^{2} 1$ and variance. Here, the hypothesis $H_{0}$ means the absence of signal and $H_{1}$ its presence. 
Performance of spectrum sensing algorithm is indicated by two metrics: by a probability of detection, $P_{d}$, which is the probability of the algorithm correctly detecting the presence of the PU and by a probability of false alarm, $P_{f a}$, which defines probability of the algorithm mistakenly declaring the presence of PU.

\subsection{Energy detection method}

Energy detection method compares the energy of the received waveform over a specified time with a threshold obtained for a given $P_{f a}$ limit to decide whether primary signal exists or not.

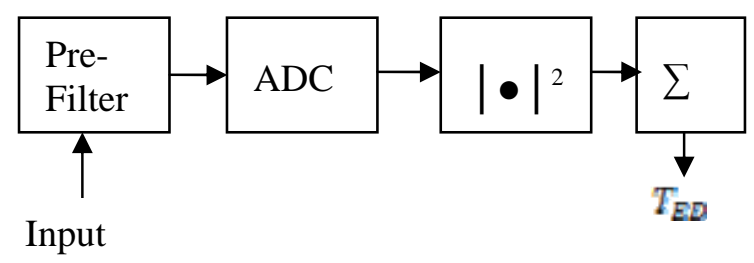

Figure 1. Implementation of energy detection

Note that for a given signal bandwidth $B$, a prefilter matched to the bandwidth of the signal needs to be applied as shown in Figure 1.

The decision statistic for energy detector is [13] [14]:

$$
T_{E D}=\frac{1}{N} \sum_{n=0}^{N-1}(|y(n)|)^{2}
$$

where $\mathrm{N}$ is the total number of taken signal samples. This decision statistic is compared with a threshold $\beta_{E D}$. If $T_{E D}>\beta_{E D}$, the signal is assumed to be present; otherwise it is assumed to be absent. In case of large $\mathrm{N}$, according to Central Limit Theorem (CLT), the probability distribution function of $T_{E D}$ can be approximated by Gaussian distribution. The expressions for probability of detection and false alarm can be obtained as:

$$
\begin{aligned}
& P_{d}=Q\left(\frac{\sqrt{N}\left(\beta_{E D}-\left(\sigma_{s}^{2}+\sigma_{\eta}^{2}\right)\right)}{\sigma_{s}^{2}+\sigma_{\eta}^{2}}\right) \\
& P_{f a}=Q\left(\frac{\sqrt{N}\left(\beta_{E D}-\sigma_{\eta}^{2}\right)}{\sigma_{\eta}^{2}}\right)
\end{aligned}
$$

where $\sigma_{s}^{2}$ is primary user signal variance, $\sigma_{\eta}^{2}$ is noise variance and $\mathrm{Q}(\mathrm{t})$ is $\mathrm{Q}$ function given by:

$$
Q(t)=\frac{1}{\sqrt{2 \pi}} \int_{t}^{+\infty} e^{-\frac{u^{2}}{2}} d u
$$

Due to the noise uncertainty [18] the estimated noise power may be different from the actual noise power by a factor $\gamma$ called as noise uncertainty factor: $\hat{\sigma}_{\eta}^{2}=\gamma \sigma_{\eta}^{2}$

The noise uncertainty bound (in $\mathrm{dB}$ ) is given as:

$A=\sup \left\{10 \log _{10} \gamma\right\}$

The noise uncertainty bound is usually 1 to 2 $\mathrm{dB}$. This creates Signal-to-Noise Ratio wall (SNR wall) under which it is not possible to detect the signal [18].The degradation in performance of ED method due to noise uncertainty is studied in detail in [19].

\subsection{Covariance Absolute Value Method}

CAV is a blind detection method which does not require any knowledge of noise and signal power, uses space-time signal correlation for signal detection. The covariances of signal and noise are generally different. The difference is used in CAV method to differentiate the signal component (PU) from background noise [15] [16].

$$
\begin{aligned}
& s(l)=[s(N-1-l), \ldots, s(-l)] \\
& \eta(l)=[\eta(N-1-l), \ldots, \eta(-l)] \\
& y(l)=[y(N-1-l), \ldots, y(-l)]
\end{aligned}
$$

where $\mathrm{N}$ is the total number of signal samples, the range of $l$ is from 0 to $\mathrm{L}-1$, the parameter $\mathrm{L}$ is called smoothing factor as explained in [15][16]. $s(l)$ represents signal samples, $\eta(l)$ represents noise samples and $y(l)$ the received signal samples.

$$
y(l)=s(l)+\eta(l)
$$

The auto-correlations of received signal is

$$
c(l)=\frac{1}{N} \sum_{n=0}^{N-1} y(n) * y(n-l) \quad l=0,1, \ldots, L-1
$$

The covariance matrix of $y(l)$ is under $H_{0}$ and $H_{1}$ is given as:

$$
\begin{aligned}
& H_{0}: R_{y}(l)=E\left[y(l) y(l)^{H}\right]=\sigma_{\eta}^{2} I_{L} \\
& H_{1}: R_{y}(l)=E\left[y(l) y(l)^{H}\right]=R_{s}(l)+\sigma_{\eta}^{2} I_{L}
\end{aligned}
$$

where $R_{s}(l)$ is the covariance matrix of $s(l)$,

$\sigma_{\eta}=E\left[\eta(l) * \eta(l)^{*}\right]$ and $I_{\mathrm{L}}$ is the identity matrix of dimension $\mathrm{L}$. If there is correlation between the signal samples due to oversampling, the $R_{s}(l)$ will be different from identity matrix. In the matrix form, the covariance matrix of the received signal is 


$$
R_{y}(N)=\left[\begin{array}{cccc}
c(0) & c(1) & \ldots & c(L-1) \\
c^{*}(1) & c(0) & \ldots & c(L-2) \\
c^{*}(L-1) & c^{*}(L-2) & \ldots & c(0)
\end{array}\right]
$$

This matrix is Toeplitz and Hermitian. Based on this covariance matrix, two metrics $T_{1}$ and $T_{2}$ are defined as follows:

$$
\begin{aligned}
& T_{1}=c(0)+\frac{2}{L} \sum_{l=1}^{L-1}(L-l)|c(l)| \\
& T_{2}=c(0)
\end{aligned}
$$

The ratio $T_{1} / T_{2}$ can be used to detect the presence of the signal. When there is no signal, $T_{1}=T_{2}$ and if the signal is present, $T_{1} / T_{2}>1$.

The following decision metric for CAV is derived as:

$$
T_{C A V}=\frac{T_{1}}{T_{2}}
$$

According to this decision metric, probability of false alarm and the probability of detection have the following form:

$$
\begin{aligned}
& P_{f a}=1-Q\left(\frac{\left.\frac{1}{\beta_{C A V}}\left(1+(L-1) \sqrt{\frac{2}{N \pi}}\right)-1\right)}{\sqrt{2 / N}}\right) \\
& P_{d}=1-Q\left(\frac{\frac{1}{\beta_{C A V}}+\frac{\beta_{L} S N R}{\beta_{C A V}(S N R+1)}-1}{\sqrt{2 / N}}\right)
\end{aligned}
$$

Signal-to-Noise Ratio is given as: $S N R=\frac{\sigma_{s}^{2}}{\sigma_{\eta}^{2}}$

, $\beta_{C A V}$ is the decision threshold and $\beta_{L}$ is a very important parameter for CAV called overall correlation coefficient. It is defined as:

$$
\beta_{L}=\frac{2}{L} \sum_{l=1}^{L-1}(L-l)|e(l)|
$$

where

$$
e(l)=\frac{E[s(n) s(n-l)]}{\sigma_{s}^{2}}
$$

This is normalized correlation strength between the signal samples (range is between 0 to 1 ).

\section{Simulation Results}

The performance of energy detector and covariance based detector is analyzed for the primary DVB-T signal, generated using MATLAB according to the ETSI specifications given in [20] intended for mobile reception of standard definition TV in 2K mode with $8 \mathrm{MHz}$ bandwidth. The received signal is sampled at the rate 36 times sampling rate at the transmitter. The SNR of the received signal is unknown. In order to use the signals for simulating the algorithms at very low SNRs, we need to add white noise to obtain various SNR levels [21]. The DVB-T signal and the added white noise are passed through a raised cosine filter with bandwidth $8 \mathrm{MHz}$, rolling factor 0.5 with 217 taps. The number of samples used is 30000 . For covariance based detection the smoothing factor is chosen as $\mathrm{L}=6$. The threshold for detection for the both methods is calculated based on $P_{f a}$. The ED without noise uncertainty is considered for comparison. Fig.2 shows the performance of ED and CAV different values of probabilities of false alarm $P_{f a}$ in AWGN channel. Both algorithms show good probability of detection for $P_{f a}=0.1$. For $P_{f a}=0.1$, ED shows probability of detection for SNR $=-20 \mathrm{~dB}$ as 0.35 compared to CAV which is 0.28 . For $P_{f a}=0.05$ and SNR $=-20 \mathrm{~dB}$, ED and CAV show probability of detection of 0.2 and 0.16 respectively. For $P_{f a}=0.01$ and SNR $=-20 \mathrm{~dB}$, ED and CAV show probability of detection of 0.17 and 0.12 respectively. Thus, ED shows the highest detection probability for different values of $P_{f a}$.

To check the performance of the methods for time- varying channels, the time-varying channel is generated based on the simplified Jakes' model with Doppler frequency $\mathrm{DF} 1=100 \mathrm{~Hz}$ and $\mathrm{DF} 2=1000 \mathrm{~Hz}$ for Rayleigh channel with $P_{f a}=0.1$ and N=30000. DF0 is no Doppler effect.

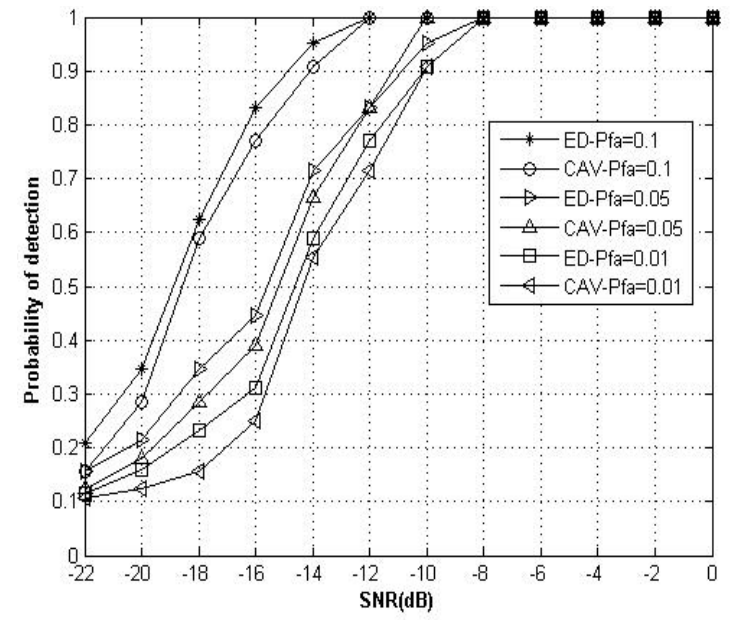

Figure 2. Analysis of probability of detection for ED and CAV for different values of $P_{f a}$ with $\mathrm{N}=30000$ in AWGN channel.

As shown in Figure 3, in case of Rayleigh fast time-varying channel at SNR of $-18 \mathrm{~dB}$, ED and CAV show probability of detection as 0.20 and 
0.15 respectively. For Rayleigh slow timevarying channel, ED and CAV show probability of detection as 0.36 and 0.28 respectively. For Rayleigh fast time-varying channel at SNR of $14 \mathrm{~dB}, \mathrm{ED}$ and CAV show probability of detection as 0.58 and 0.52 respectively.

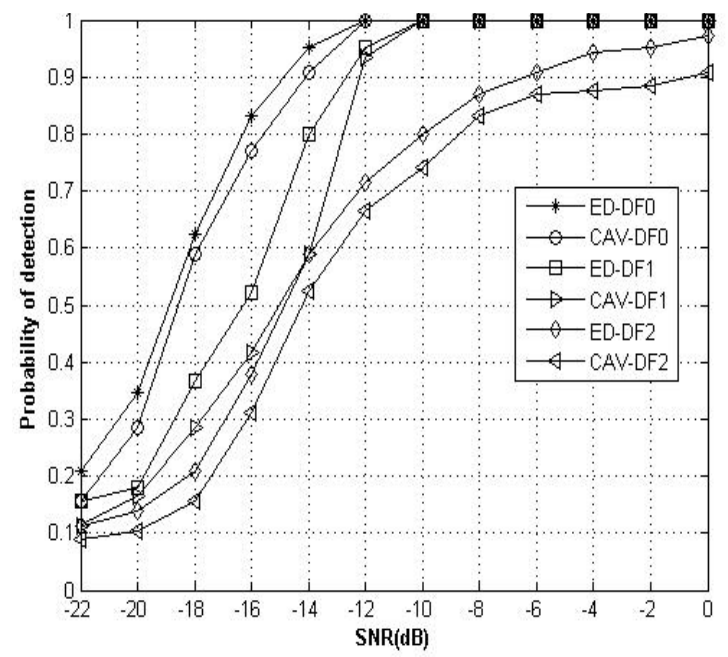

Figure 3. Analysis of probability of detection for ED and CAV with $P_{f a}=0.1, \mathrm{~N}=30000$ under Rayleigh time- varying channel.

For Rayleigh slow time-varying channel at SNR of $-14 \mathrm{~dB}, \mathrm{ED}$ and CAV show probability of detection as 0.8 and 0.58 respectively. This clearly reveals that the degradation in performance for fast timevarying channel is more compared to slow timevarying channel. The performance of CAV degrades more as compared to ED in Rayleigh fast time varying channel.

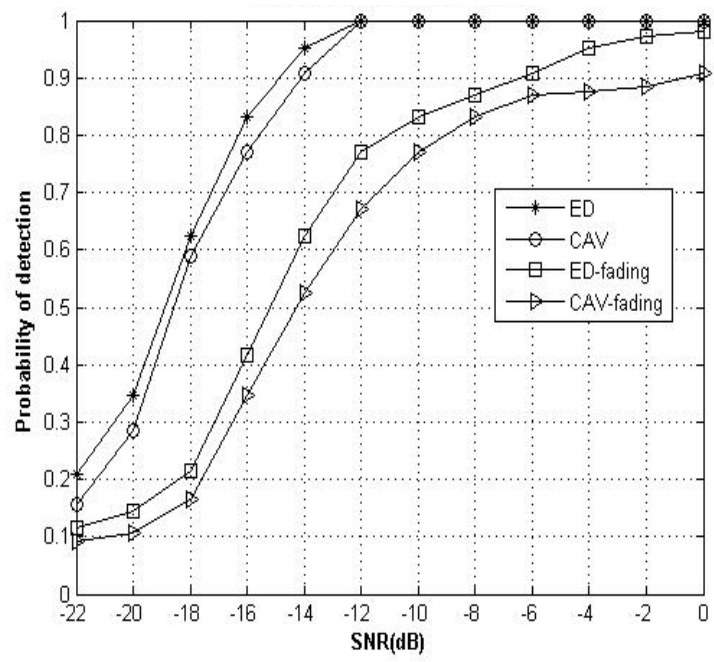

Figure 4. Analysis of probability of detection for ED and CAV for $P_{f a}=0.1$ and $\mathrm{N}=30000$ under

Rician time-varying channel with $\mathrm{K}=1$.

For Rician channel, Doppler frequency of $100 \mathrm{~Hz}$ with $P_{f a}=0.1$ and $\mathrm{N}=30000$ is considered, Figure 4 and Figure 5 reflect its effect on the probability of detection for ED and $\mathrm{CAV}$ for $\mathrm{K}=1$ and $\mathrm{K}=10$. For $\mathrm{K}=1$ and $\mathrm{SNR}=-14 \mathrm{~dB}$, ED and CAV show probability of detection of 0.62 and 0.52 , respectively. For $\mathrm{K}=10$ and $\mathrm{SNR}=-14 \mathrm{~dB}$, ED and CAV show probability of detection of 0.83 and 0.58 , respectively. The performance of both methods degrades, but the degradation is more with $\mathrm{K}=1$ compared to $\mathrm{K}=10$.

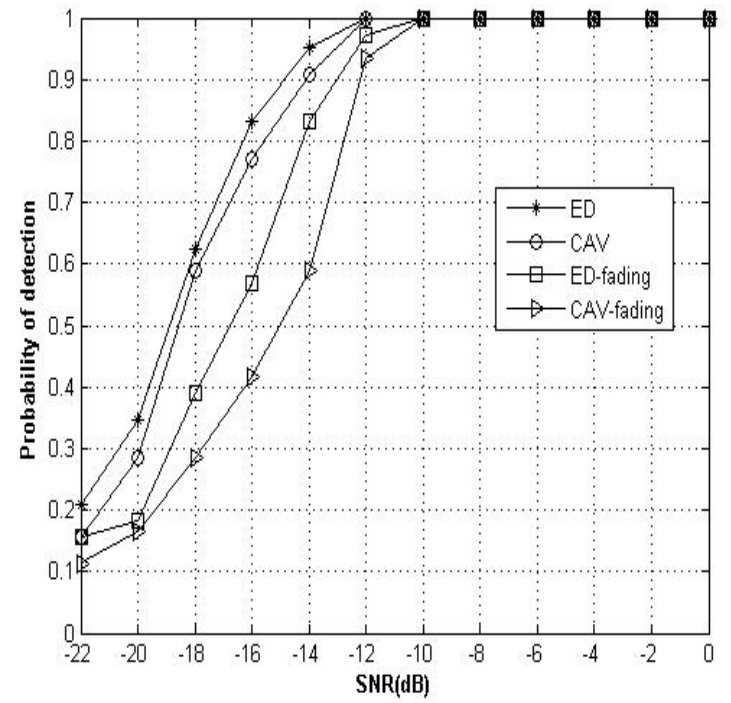

Figure 5. Analysis of probability of detection for ED and CAV for $P_{f a}=0.1$ with $\mathrm{N}=30000$ under Rician time-varying channel with $\mathrm{K}=10$.

The effect of time-varying channels at Doppler frequency of $100 \mathrm{~Hz}$ on the ROC curve is analyzed for both the methods by plotting Fig.6 and Figure 7 with values $\mathrm{SNR}=-14 \mathrm{~dB}$, $\mathrm{N}=30000$ and $P_{f a}=0.1$.Fig. 6 reflects ROC curve under Rayleigh channel. There is performance deterioration for the both: ED and CAV, but ED provides probability of detection better than CAV. Fig.6 reflects ROC curve under Rician channel with $K=1$, which shows performance deterioration for the both: ED and CAV at SNR of $-14 \mathrm{~dB}$ compared to $\mathrm{K}=10$. But if the curves with $K=1$ and $K=10$ are compared, the performance of detection schemes are better for $K=10$. Figure 6 and Figure 7 reveal that ROC curve will be poor at SNR values lower than $-14 \mathrm{~dB}$. 


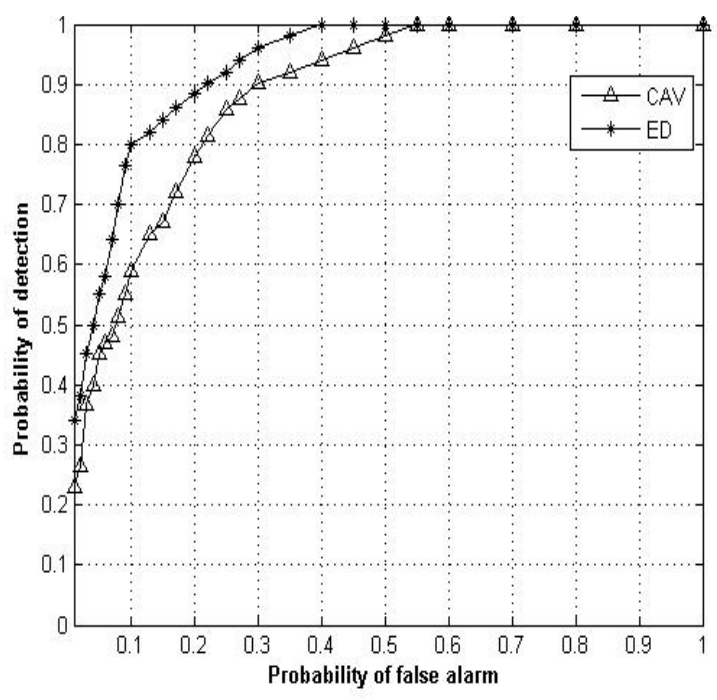

Figure 6. ROC curve for ED and CAV with $P_{f a}$ $=0.1, \mathrm{SNR}=-14 \mathrm{~dB}$ and $\mathrm{N}=30000$ under Rayleigh time-varying channel.

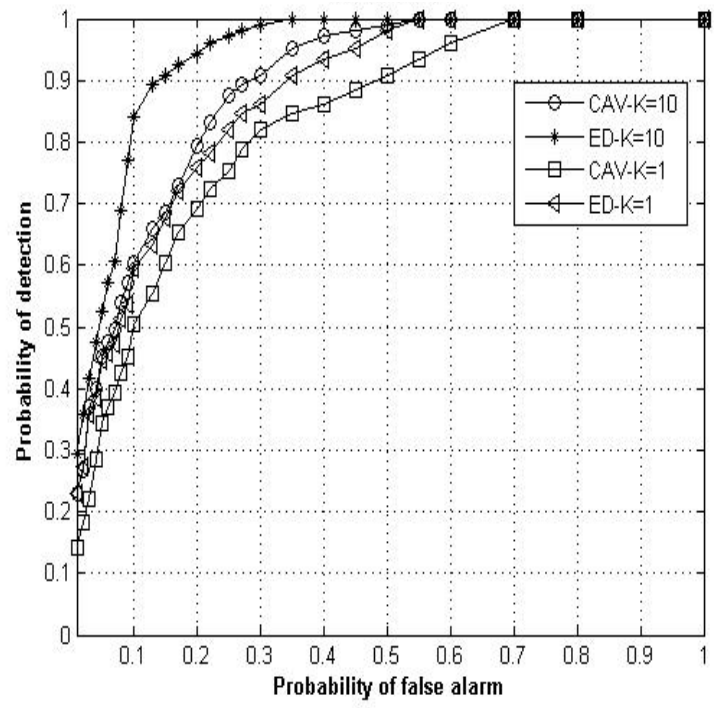

Figure 7. ROC curve for ED and CAV with $P_{f a}$ $=0.1$, SNR $=-14 \mathrm{~dB}$ and $\mathrm{N}=30000$ under Rician timevarying channel for $\mathrm{K}=1$ and $\mathrm{K}=10$.

To test the impact of smoothing factor, we fix $P_{f a}=0.1, \mathrm{SNR}=-20 \mathrm{~dB}$ and $\mathrm{N}=30000$ and vary smoothing factor L from 5 to 10. Fig.8 shows the results for probability of detection. Since ED is not dependent on $\mathrm{L}$, there is a line parallel to X-axis. For CAV, as L increase from 5 to 9 , the probability of detection increases from 0.22 to 0.75 . The probability of detection is not very sensitive for smoothing factor greater than 9 . It can be noted that smaller the value of $\mathrm{L}$ means lower complexity in practice; thus, we can select a relatively small $\mathrm{L}$. However, it is difficult to choose the best smoothing factor so that computational complexity reduces as it is related to the signal property which is unknown.

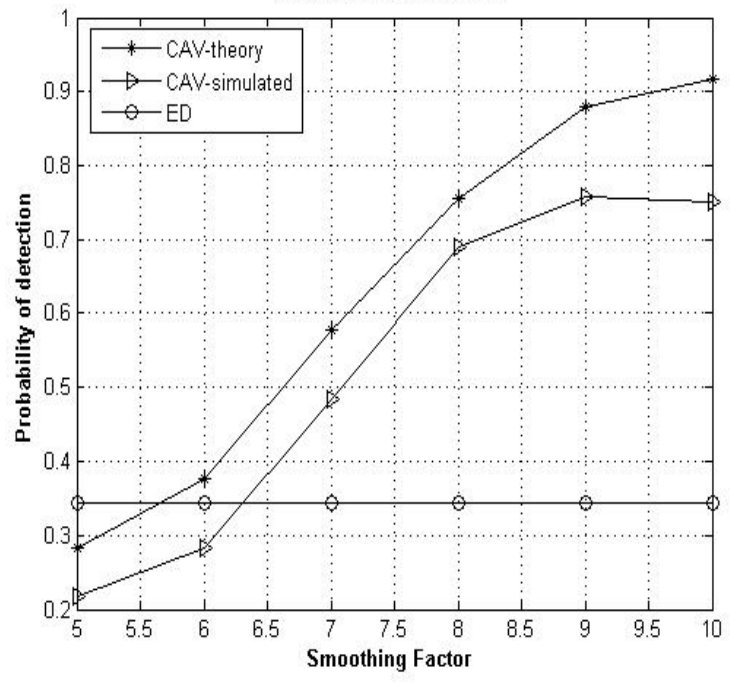

Figure 8. Probability of detection versus smoothing factor for $P_{f a}=0.1, \mathrm{SNR}=-20 \mathrm{~dB}$ and $\mathrm{N}=30000$.

For testing the impact of overall correlation coefficient on probability of detection for CAV, the values of $P_{f a}=0.1$ and $\mathrm{SNR}=-14 \mathrm{~dB}$ and $\mathrm{N}=30000$ are fixed. With "ED-x dB" means energy detection with $\mathrm{x}$ - $\mathrm{dB}$ noise uncertainties. As the overall correlation coefficient increases, the probability of detection also increases. Figure 9 reveals this fact. The theoretical probability of detection for CAV is also plotted. Further the probability of detection for ED and ED-0.4 dB are plotted. It clearly reveals that ED and ED-0.4dB are not dependent on correlation coefficient so there is line parallel to $\mathrm{x}$-axis. ED with $0.4 \mathrm{~dB}$ noise uncertainty shows poor probability of detection.

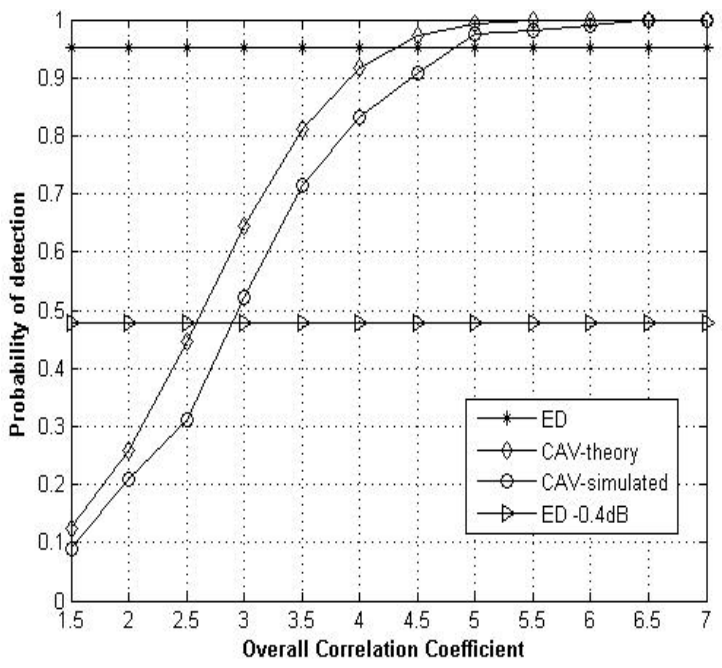

Figure 9. Probability of detection versus overall correlation coefficient for $P_{f a}=0.1, \mathrm{SNR}=-14 \mathrm{~dB}$ and $\mathrm{N}=30000$. 


\section{Conclusions}

In this paper, sensing algorithms based on energy detection without noise uncertainty and covariance based detection is analyzed. The simulation results show that energy detection can be applied for signal detection with a priori noise power knowledge and covariance method can be applied for signal detection application without knowledge of signal, channel and noise power. The simulation based on DVB-T signals has been carried out to evaluate the performance of both methods. The performance of energy detection and covariance based spectrum sensing degrades in fast time-varying fading channels. This degradation in performance occurs less in case of slow timevarying channels. The energy detection without noise uncertainty outperformed the covariance based method in case of fading time-varying channels. The probability of detection for covariance based detection improves as smoothing factor and overall correlation coefficient increase. ED with $0.4 \mathrm{~dB}$ noise uncertainty shows poor probability of detection. This analysis provides a new insight in spectrum sensing for dynamic spectrum access in cognitive radio network and can be applied to IEEE 802.11af standard for spectrum sensing exploiting the TV white space.

\section{REFERENCES}

1. FCC, Spectrum policy task force report, in Proceedings of the Federal Communications Commission (FCC '02), Washington, DC, USA, Nov. 2002.

2. MITOLA J., G. Q. MAGUIRE, Cognitive Radio: Making Software Radios more Personal, IEEE Personal Communication, August 1999, vol. 6, no. 4, pp 13-18.

3. HAYKIN, S., Cognitive Radio: Brainempowered Wireless Communications, IEEE Transactions Communications, February 2005, vol. 23, no. 2, pp. 201-220.

4. DHOPE, T., D. SIMUNIC, M. DJUREK, Application of DOA Estimation Algorithms in Smart Antenna Systems, Studies in informatics and Control, Dec. 2010, vol.19, Issue 4, pp. 445-452.

5. CHEN, K-C., R. PRASAD, Cognitive Radio Networks, John Wiley \& Sons Ltd, 2009.
6. 802.11 Working Group, IEEE P802.11af D0.06: Draft Standard for Information Technology, Oct.2010, http//www.ieee802.org/11/

7. http://www.wpc.dot.gov.in/DocFiles/Revis ed NFAP Meeting Schedule.pdf

8. http://www.wpc.dot.gov.in/DocFiles/Draft Channelling Plan for NFAT-2011

9. DHOPE, T., D. SIMUNIC, R. PRASAD, TVWS Opportunities and Regulation:Empowering Rural India, $14^{\text {th }}$ International Symposium on WPMC '11, Brest, France, Oct. 2011, pp. 201-205.

10. DHOPE, T., D. SIMUNIC, R. PRASAD, TVWS Opportunities and Regulation:Empowering Rural India, $14^{\text {th }}$ International Symposium on WPMC '11, Brest, France, Oct. 2011, pp. 566-570.

11. ZENG, Y. H., Y. C. LIANG, A. T. HOANG, R. ZHANG, Review on Spectrum Sensing for Cognitive Radio: Challenges and Solutions, EURASIP Journal on Advances in Signal Processing, 2010, Vol. 2010, pp. 1-15.

12. URKOWITZ, H., Energy Detection of Unknown Deterministic Signals, Proceedings of the IEEE, 1967, vol. 55, no. 4, pp. 523-531.

13. DHOPE, T., D. SIMUNIC, R. PRASAD, Hybrid Detection Method for Cognitive Radio, $13^{\text {th }}$ International conferenace on SoftCOM SoftCOM2011, Split-HvarDubrovnik, Sept. 2011, SS1-78741-1609.

14. CABRIC, D., A. TKACHENKO, R. W. BRODERSEN, Experimental Study of Spectrum Sensing Based on Energy Detection and Network Cooperation, in Proc. of the ACM International Workshop on Technology and Policy for Accessing Spectrum, Baltimore, MD. Aug. 2006.

15. ZENG, Y. H., Y.-C. LIANG, SpectrumSensing Algorithms for Cognitive Radio Based on Statistical Covariances, IEEE Transactions on Vehicular Technology, 2009, vol. 58, no. 4, pp. 1804-1815.

16. ZENG, Y. H., Y.-C. LIANG, Covariance Based Signal Detections for Cognitive Radio, DYSPAN07, Ireland, April 2007, pp. 202-207. 
17. DHOPE, T., D. SIMUNIC, Hybrid Detection Method for Spectrum Sensing in Cognitive Radio, (in press) $35^{\text {th }}$ Jubilee International Convention MIPRO 2012, May 2012.

18. TANDRA, R., A. SAHAI, Fundamental limits on detection inlow SNR under noise uncertainty, inWirelessCom 2005, Maui, HI, vol. 1, June 2005, pp. 464-469.

19. DHOPE, T., D. SIMUNIC, Performance Analysis of Covariance Based Detection in Cognitive Radio, (in press) $35^{\text {th }}$ Jubilee International Convention MIPRO 2012, May 2012
20. http://www.etsi.org/DVB-Terristerial Standards.

21. SHELLHAMMER, S. S., G. CHOUINARD, M. MUTERSPAUGH, M. GHOSH, Spectrum Sensing Simulation Model, [Online]. Available: http://grouper.ieee.org/groups/802/22/, Jul.2006. 